POS PROCEEDINGS

\title{
Experiments at the VEPP-2000 e+e- Collider
}

\section{S. I. Serednyakov*}

Novosibirsk State University

Budker Institute of Nuclear Physics, SB RAS,Novosibirsk, Russia

E-mail: seredn@inp.nsk.su

Beginning from 2011, experiments are carrying out at the low energy $e^{+} e^{-}$collider VEPP-2000 in the energy range $0.3 \div 2.0 \mathrm{GeV}$. New results on $e^{+} e^{-} \rightarrow$ hadrons cross sections are presented, including $e^{+} e^{-} \rightarrow \pi^{+} \pi^{-}, K^{+} K^{-}, \pi^{+} \pi^{-} \pi^{0}, \eta K^{+} K^{-}, p \bar{p}, n \bar{n}$ etc $\ldots$ Applications of the obtained results are discussed.

XXVII International Symposium on Lepton Photon Interactions at High Energies

17-22 August 2015

Ljubljana, Slovenia

* Speaker.

$\dagger$ 
Experiments. The collider VEPP-2000 [1] (Fig.1) is intended for $e^{+} e^{-}$experiments in the center of mass (c.m.) energy range $E=0.3-2.0 \mathrm{GeV}$. In comparison with its predecessor VEPP$2 \mathrm{M}$ [2] it has higher energy and higher luminosity up to $10^{31} \mathrm{~cm}^{-2} \mathrm{sec}^{-1}$ at $2 \mathrm{GeV}$. Two detectors

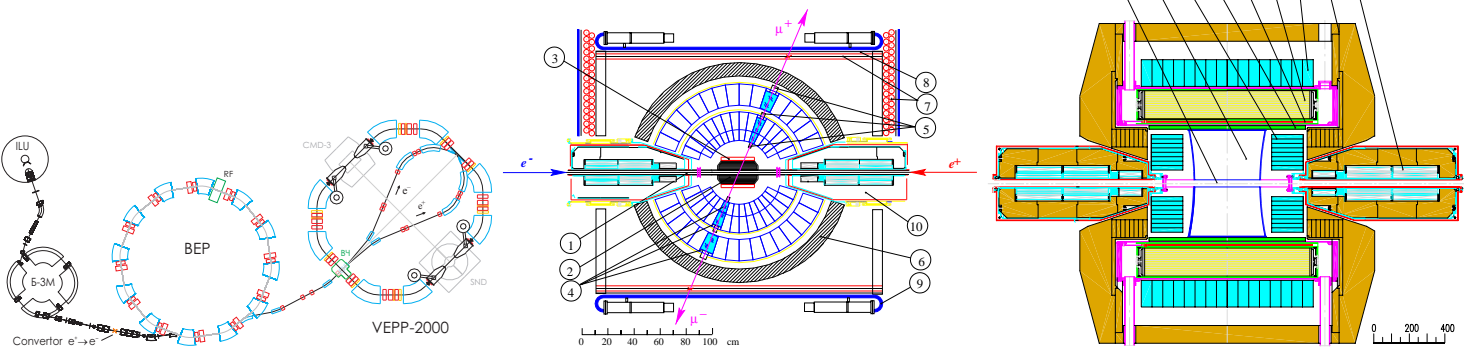

Figure 1: Left: VEPP-2000 collider. ILU - 3 MeV linear accelerator, B-3M - $200 \mathrm{Mev}$ electron synchrotron, BEP - booster for electrons and positrons. Middle: SND detector. 1 - beam pipe, 2 - tracker, 3 - aerogel Cherenkov counter, 4 - NaI(Tl) EMC, 5 - photodetectors, 6 - iron, 7-9 - muon detector, 10 - SC solenoides. Right: CMD3 layout. 1 - beam pipe, 2 - tracker, 3 - BGO EMC, 4 - Z-chamber, 5 - magnet coil, 6 - LXE EMC, 7 - CsI(Tl) EMC, 8 - yoke, 9 - collider solenoid. $\mu$-system is not shown.

SND [3] and CMD-3 [4], shown in Fig.1, perform experiments at VEPP-2000.
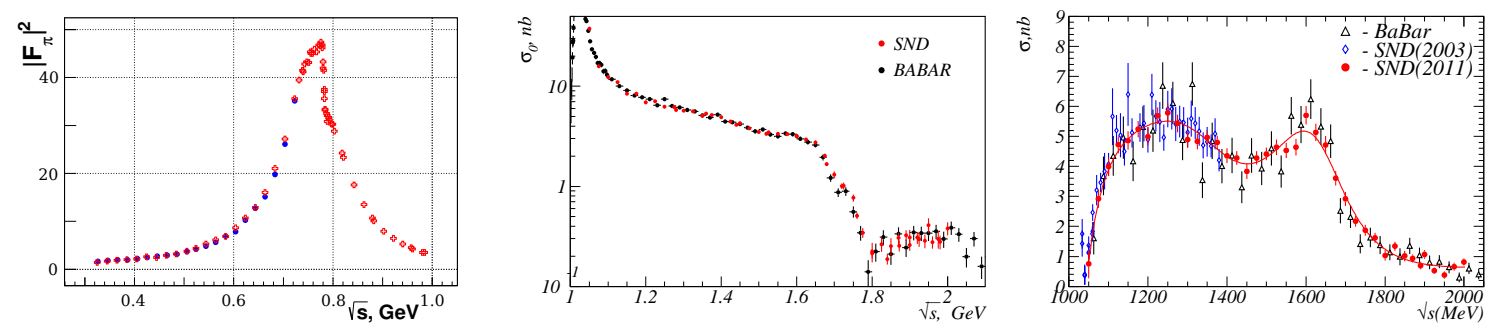

Figure 2: Left: The pion electromagnetic form factor, measured by CMD-3 [12]. Middle: The cross section of the $e^{+} e^{-} \rightarrow K^{+} K^{-}$process, measured by SND. Right: The cross section of the $e^{+} e^{-} \rightarrow \pi^{+} \pi^{-} \pi^{0}$ process from SND.

The physical program for VEPP-2000 includes precise measurements of all major channels of $e^{+} e^{-}$annihilation to hadrons and hence the value $\mathrm{R}=\sigma\left(e^{+} e^{-} \rightarrow\right.$ hadrons $) / \sigma\left(e^{+} e^{-} \rightarrow \mu^{+} \mu^{-}\right)$used in calculation of the muon anomaly $(g-2)_{\mu}$ and the fine structure constant at Z-mass $\alpha_{e m}\left(s=M_{Z}^{2}\right)$. Other items of the program are: the comparison of the $e^{+} e^{-} \rightarrow$ hadrons isovector cross sections with the $\tau$ decay spectra; study of the production and decays of the excited vector states $\rho \prime_{s}, \omega \prime_{s}, \phi \prime_{s}$; study of the nucleons production at the threshold.

In the series of runs in 2010-2013 each detector accumulated about $70 \mathrm{pb}^{-1}$ of data. Some physical results are published already $[5,6,7,8,9,10,11]$. In this talk recent SND and CMD-3 results are presented.

Productions of mesons. The $e^{+} e^{-} \rightarrow \pi^{+} \pi^{-}$cross section is measured by CMD-3 (Fig.2) using $\sim 10^{7}$ events. The systematic accuracy is limited mainly by $e / \pi / \mu$ separation, fiducial volume determination, beam energy measurement and radiative correction calculation. Its total value is expected to be $\sim 0.3 \%$ [12]. The process $e^{+} e^{-} \rightarrow K^{+} K^{-}$is studied by SND (Fig.2). Charged kaons are identified by the aerogel Cherenkov counter [13] and $d E / d x$ measurements in 
drift chamber. The complex cross section energy dependence in Fig.2 is due to the contribution of $\rho^{\prime}, \rho^{\prime \prime}, \omega \prime, \omega \prime \prime, \phi \prime$ resonances and their interference. Our data agree with previuos Babar results [14]. The systematics uncertainty is estimated to be $2-3 \%$. The isocalar process $e^{+} e^{-} \rightarrow \pi^{+} \pi^{-} \pi^{0}$ has been studied by SND above $1.05 \mathrm{GeV}$ [15]. The cross section (Fig.2) is entirely described by two excited $\omega$-like states: $\omega(1420)$ and $\omega(1650)$. The $e^{+} e^{-} \rightarrow \eta K^{+} K^{-}$cross section, measured by CMD-3 [12] (Fig.3), agrees well with earlier measurements. The $e^{+} e^{-} \rightarrow \eta \pi^{+} \pi^{-} \pi^{0}$ cross section, measured by SND for the first time, is shown in Fig.3. The $\omega(1680)$ and $\phi(1650)$ states give the major contribution in this process.
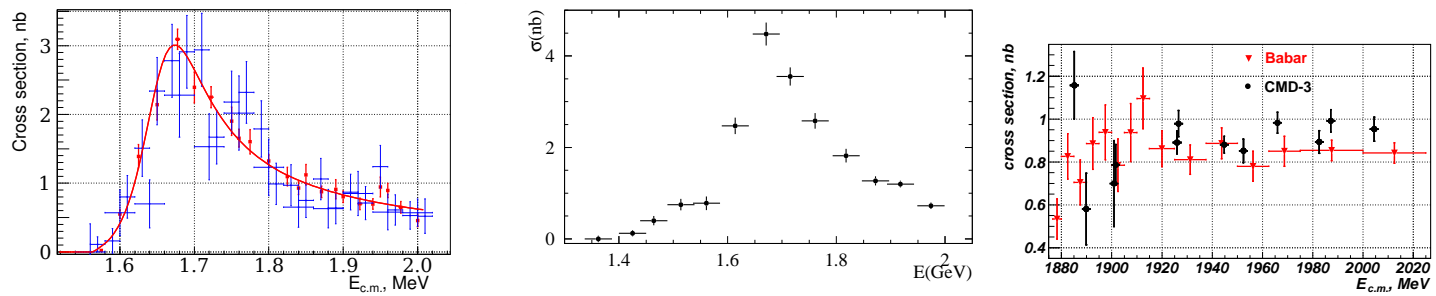

Figure 3: Left: The cross section of the $e^{+} e^{-} \rightarrow \eta K^{+} K^{-}$process, measured by CMD-3. Middle: The cross section of the $e^{+} e^{-} \rightarrow \eta \pi^{+} \pi^{-} \pi^{0}$ process from SND. Right: The cross section of the $e^{+} e^{-} \rightarrow p \bar{p}$ process, measured by CMD-3.

Production of nucleon-antinucleon pairs. The $e^{+} e^{-} \rightarrow p \bar{p}$ process was studied in many experiments, most accurate is of Babar [16]. SND and CMD-3 preliminary data agree well with Babar [17] (Fig.3). Regarding the proton $\left|G_{E} / G_{M}\right|$ ratio, CMD-3 definitely confirms the Babar result that $\left|G_{E} / G_{M}\right|>1$ (Fig.4). The $e^{+} e^{-} \rightarrow n \bar{n}$ cross section measured by SND [8] does slowly varies in the threshold region (Fig.4), and its value is close to that for $e^{+} e^{-} \rightarrow p \bar{p}$. SND cross section agrees with the FENICE measurement [18] but more precise.
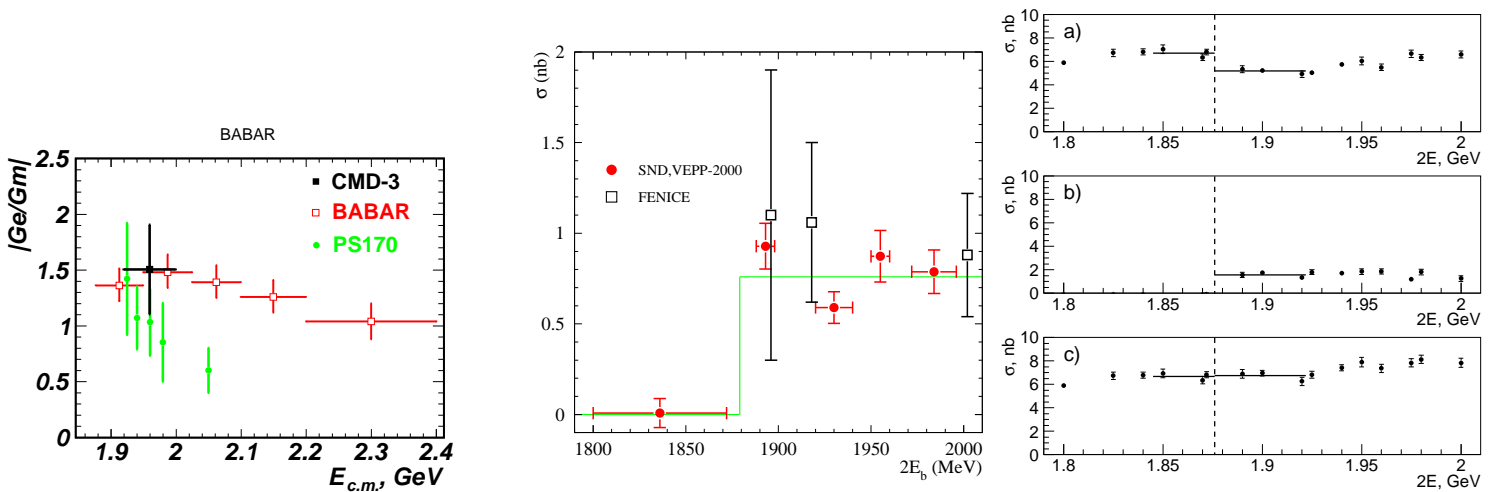

Figure 4: Left: The ratio $\left|G_{E} / G_{M}\right|$ for proton measured by CMD-3 in comparison with earlier data. Middle: The cross section of the $e^{+} e^{-} \rightarrow n \bar{n}$ process [8] measured by SND. Right: The cross sections near the nucleon-antinucleon threshold. (a) process $e^{+} e^{-} \rightarrow 6 \pi$, (b) processes $e^{+} e^{-} \rightarrow p \bar{p}, n \bar{n}$, (c) sum of a) and b).

The sum cross section of $e^{+} e^{-} \rightarrow p \bar{p}$ and $e^{+} e^{-} \rightarrow n \bar{n}$ has the step-like shape with step height of about $1.7 \mathrm{nb}$ (Fig.4,right). One can expect that such a step in the nucleon-antinucleon cross section must be compensating by a similar negative step in the meson production cross section. It turns out that such $1.7 \mathrm{nb}$ negative step is observed in the $e^{+} e^{-} \rightarrow 6 \pi$ channel [6], while the 
full cross section (sum of $e^{+} e^{-} \rightarrow 6 \pi$ and $e^{+} e^{-} \rightarrow p \bar{p}, n \bar{n}$ ) has no structure (Fig.4). Now there is no explanation, why only $e^{+} e^{-} \rightarrow 6 \pi$ channel is sufficient to compensate the nucleon-antinucleon step.

Conclusions The $e^{+} e^{-}$coliider VEPP-2000, operating in the energy range $0.3 \div 2.0 \mathrm{GeV}$, and two detectors CMD-3 and SND, are shortly described. The results of analysis of processes $e^{+} e^{-} \rightarrow \pi^{+} \pi^{-}, K^{+} K^{-}, \pi^{+} \pi^{-} \pi^{0}, \eta K^{+} K^{-}, \eta \pi^{+} \pi^{-} \pi^{0}, p \bar{p}, n \bar{n}$ are presented. The physical program and significance of new data are discussed.

Acknowledgment. The author expresses his gratitude to S.I. Eidelman, V.P. Druzhinin and V.F. Kazanin for fruitfull discussion. This work is partially supported in the framework of the State order of the Russian Ministry of Science and Education and the RFBR grants No. 15-02-01037 and Sci.School 2479.2014.2.

\section{References}

[1] Yu. M. Shatunov et al., in Proceedings of the 7th European Particle Accelerator Conference, Vienna, 2000 (EPS, Geneva, 2000), p. 439. [http://accelconf.web.cern.ch/AccelConf/e00/PAPERS/MOP4A08.pdf].

[2] I.A.Koop et al., Publised in: Physics and detectors for DAPHNE, Frascati (1999) 393-404.

[3] M. N. Achasov et al., Nucl. Instrum. Methods Phys. Res., Sect. A 449, 125 (2000).

[4] B. I. Khazin, Nucl. Phys. B. Proc. Suppl. 181-183, 376 (2008)

[5] M. N. Achasov et al., Phys. Rev. D, 88, 054013 (2013)

[6] A. E. Obrazovsky, S. I. Serednyakov, JETP. Lett. 99, 363 (2014)

[7] M. N. Achasov et al., Phys. Rev. D, 90, 032002 (2014)

[8] M. N. Achasov et al., Phys. Rev. D, 90, 112007 (2014)

[9] V. M. Aulchenko et al., Phys. Rev. D, 91, 052013 (2015)

[10] R. R. Akhmetshin et al., Phys. Lett., B723, 82 (2013)

[11] R. R. Akhmetshin et al., Phys. Lett., B740, 273 (2015)

[12] V. F. Kazanin, Talk given at PHOTON-2015 Conference, 15-19 June 2015, Novosibirsk

[13] A. Yu. Barnyakov et al. Nucl. Instr. Meth. A732, 330 (2013)

[14] J. P. Lees et al. (Babar Collaboration), Phys. Rev. D 88, 032013 (2013)

[15] V. M. Aulchenko et al. JETP, 121, No.1, 27-34 (2015)

[16] J. P. Lees et al. (Babar Collaboration), Phys. Rev. D 87, 092005 (2013)

[17] R. R. Akhmetshin, arXiv:1507.08013 [hep-ex]

[18] A. Antonelli et al., (FENICE Collaboration), Nucl.Phys. B517, 3 (1998) 